\title{
Bioanalysis
}

\section{Incurred sample reproducibility and stability assessment in a cell-based drug concentration assay}

\begin{abstract}
Joleen White is Principal Scientist in Translational Sciences at Biogen Idec. Throughout her career, she has applied her background in biophysical protein chemistry to pharmaceutical development in therapeutic indications with significant unmet medical need. In her current role, she supports method development and regulated bioanalysis of biomarkers, biopharmaceuticals, and immunogenicity in biological samples from nonclinical and clinical studies. Her experience with measuring macromolecules includes enzymes, monoclonal antibodies, Fc fusions, oligonucleotides, PEGylated proteins, and other novel protein constructs. She has supported studies from discovery through all phases of development including GLP nonclinical, clinical, and post-marketing commitments.
\end{abstract}

Incurred sample reproducibility is one aspect of in-study validation, with white papers outlining expectations for chromatographic assays and immunoassays. This manuscript outlines an approach for performing incurred sample reproducibility for a bioequivalence study using a cell-based assay, with the complication of time elapsed between original and repeat assays. The incurred sample reproducibility passed the pre-established acceptance criteria of $45 \%$ for at least $2 / 3$ of the samples: $174 / 216$ samples (80.6\%). Data trends between the two crossover arms were qualitatively similar. The passed incurred sample reproducibility and stability further supports the validity of the original study conclusion that the two manufacturing processes were bioequivalent. This illustrates one approach to extrapolating industry and regulatory recommendations for situations outside current guidance.

A primary goal of the bioanalytical scientist is to provide valid and robust data to support the science-driven drug development decisions. When measuring drug concentration for clinical studies, preanalytical characterization of assay performance is complemented by in-study validation to ensure that assay performance is maintained. In recent years, incurred sample reproducibility (ISR) analysis has been widely adopted by the bioanalytical community as a critical in-study validation requirement [1-4]. Bioequivalence studies are expected to conduct ISR regardless of whether it has previously been conducted in that species and matrix. In addition, during the testing phase, samples from subjects in both arms of the bioequivalence study are typically integrated across analytical runs to ensure that intra-assay precision does not adversely affect interpretation. The conduct of the study and in-study assay validation experiments, including ISR, help ensure the absence of bias between the two study arms.

This manuscript focuses on the bioanalytical challenge of conducting ISR for a previously validated cell-based assay. The cell-based assay format is neither explicitly described in regulatory guideline or guidance nor in the industry white papers. To demonstrate that assay performance in-study matches the prestudy validation performance, this manuscript describes designing the ISR experiment, setting appropriate



Joleen T White

Biogen, 250 Binney Street,

Cambridge, MA 02142, USA

Currently at EMD Serono, 45A Middlesex

Turnpike, Billerica, MA 01821

\section{Mary Crossman}

Author for correspondence: Biogen, 250 Binney Street, Cambridge, MA 02142, USA mary.crossman@biogen.com

Meena Subramanyam Biogen, 250 Binney Street, Cambridge, MA 02142, USA 


\section{Key terms}

Bioequivalence: Evaluation of the PK and/or PD

similarity between two different lots or manufacturing

processes, sometimes referred to as biocomparability for a

complicated biotherapeutic.

Incurred sample reproducibility: Evaluation of the reproducibility of test results for incurred samples with unknown nominal results, typically conducted concurrent with sample testing phase.

acceptance criteria and evaluating the data. This case study provides an illustrative example of applying scientific principles to extrapolate from prior work and guidelines to novel scenarios.

The proposed ISR acceptance criteria for chromatographic assays and immunoassays in white papers and regulatory guidance are based on the QC assay acceptance criteria for accuracy and precision [2]. In this context, however, it is important to keep in mind that the ISR acceptance criteria for individual assays should match the intended purpose of the assay. In this case, the accuracy and precision of the method in validation was greater than the typical targets for immunoassays. Since this accuracy and precision was suitable for the intended purpose, and the ISR is intended to show that the assay performance in study is similar to validation, the ISR acceptance criteria were modified accordingly. As the white papers [1-2], EMA guideline [3] and draft FDA guidance [4] are nonbinding documents to inform the public and industry about the industry and regulatory agency thinking on this topic, we used the same scientific principles to conduct the ISR investigation for this cell-based bioassay.

In this case study of Drug X, a cytokine therapeutic, a cell-based drug concentration assay was selected to measure the serum concentration as it was the only format with sufficient sensitivity to characterize the full PK profile in a human pharmacology study to evaluate the bioequivalence of manufacturing processes $\mathrm{A}$ and $\mathrm{B}$. As previously observed in other quantitative drug concentration methods [5], cell-based assays are typically less precise than immunoassays due to the use of living cells as a critical reagent. Although this cell-based format was optimized to maximize sensitivity and minimize imprecision, the final accuracy and precision acceptance criterion was $\pm 25 \%$ (30\% at LLOQ). For the intended purpose, this precision was an acceptable trade-off to ensure that the sensitivity requirement was met.

The ISR was not conducted at the time of original sample analysis from the bioequivalence study, due to evolving regulatory and industry recommendations. The ISR was conducted nearly 2 years later to comply with regulatory expectations, near the end of pre-established spiked QC stability. Due to the elapsed time, the ISR experiment represents a blended in-study validation experiment of both reproducibility and stability. Although there is no scientific reason to suspect differential stability of spiked QCs and incurred samples for Drug X [6], nearly 2 years between analyses did exceed any prior history with this assay. Since this ISR experiment was recognized as a blended design between ISR and stability of incurred samples, acceptable results would imply that both assessments passed, A potential failure would be confounded by the possibility that one assessment passed while the other failed.

Additional practical complications were also noted prior to initiating analysis, and were mitigated as much as feasible.

- Per the internal standard operating practice, the original aliquot used to derive the primary concentration result was discarded after finalizing the clinical study report, so the ISR had to be performed on the backup aliquot. Although there is no direct mitigation for this challenge, the variability between aliquots was anticipated to be random;

- Multiple reagent lots were necessarily different and the analyst who originally analyzed the samples was no longer employed at the analytical lab. To mitigate this challenge, the original QCs used during initial sample analysis were located and used to bridge the current reagent lots and analysts. In addition, a sufficient aliquot number of the original QCs were available to use as the analytical QCs for ISR sample analysis;

- Because the concentration profiles were close to the LLOQ, concentration results for a large number of samples were below the LLOQ, and approximately half of the numerical results were below the threshold of $3 \times$ LLOQ recommended for sample selection in the ISR guidance. To include more subjects in the ISR sample set, a threshold of $2 \times$ LLOQ was used for sample selection, with roughly $2 / 3$ of the samples having results above $3 \times$ LLOQ.

Taking into account the above challenges, the combined ISR and stability experiment was carefully designed and performed to evaluate whether the sample concentration results derived from this assay were valid to support the original study conclusion of bioequivalence between manufacturing processes $\mathrm{A}$ and $\mathrm{B}$. The incurred sample reproducibility passed the pre-established acceptance criteria of $45 \%$ for at least $2 / 3$ of the samples: $174 / 216$ samples (80.6\%). Data trends between the two crossover arms were qualitatively similar. The 
passed incurred sample reproducibility and stability further supports the validity of the original study conclusion that the two manufacturing processes were bioequivalent. This illustrates one approach to extrapolating industry and regulatory recommendations for situations outside current guidance.

\section{Methods}

\section{Cell-based drug concentration assay}

Cells expressing Receptor $\mathrm{Y}$ were plated overnight. Sera samples and controls diluted in culture medium were incubated with the cells. Drug $X$ in the samples bound to Receptor Y and elicited a cascade of biological events, including increased expression of Protein Z. Cell lysates were tested in an immunoassay detecting Protein Z, using additional QCs for Protein Z concentration in addition to the Drug X QCs included in the cell-based assay step.

Prestudy validation accuracy and precision were evaluated with three replicates of five spiked QC levels on seven plates, and calculated as previously described [7]. The intra-assay precision $\left(\% \mathrm{CV}_{\mathrm{p}}\right)$ was $\leq 13.2 \%$ (19.5\% for LLOQ), the interassay precision $\left(\% \mathrm{CV}_{\mathrm{t}}\right)$ was $\leq 18.4 \%$ (19.5\% for LLOQ) and the absolute value of interassay accuracy (\%RE) was $\leq 7.4 \%$ (4.1\% for LLOQ). Across these runs, $101 / 105$ individual QCs passed the analytical acceptance criteria and all seven runs passes the aggregate analytical acceptance criteria.

Spiked QC samples were used to establish longterm stability up to 24 months at $-70^{\circ} \mathrm{C}$ to $-90^{\circ} \mathrm{C}$. The low and high QC levels were run in triplicate at each stability time point against fresh standard curves and fresh analytical QCs. Time points at 1, 3, 6, 9, 12, 18, 21 and 24 months had minimally $2 / 3$ stability QCs within acceptance criteria at each time point, and no downward trend in analytical recovery (measured concentration/ nominal concentration).

\section{Sample selection for incurred sample reanalysis \& stability}

The clinical study was a bioequivalence study with a cross-over design between manufacturing processes $\mathrm{A}$ and $\mathrm{B}$. Half the subjects started with each manufacturing process. Protocols and informed consent forms were approved by the appropriate institutional review board for each site, the study was conducted according to International Conference on Harmonization Guidelines for Good Clinical Practice and the Declaration of Helsinki, and all patients provided written informed consent before entering the study [8-9].

The incurred study samples selected for ISR were based on adapting the industry recommendations to this particular assay, plus considering the intended purpose of the study and the practical limitations of sample availability. Due to the low concentrations measured in the original testing phase, individual sera samples were selected that had reported results $\geq 2 \times$ LLOQ, with about two-thirds $\geq 3 \times$ LLOQ. A minimum of 100 individual samples were selected plus 5\% of the samples greater than 1000 , resulting in 216 of 3315 samples (6.5\% of total study samples).

Since the purpose of the ISR was to ensure that there were no concerns with the bioanalytical data used in the bioequivalence assessment, no samples were included from any subjects that were excluded from the bioequivalence evaluation for any reason. As a conservative sample number estimate, samples that were below the LLOQ were included in the denominator to determine the number of samples to test, although these samples are often excluded for first in human trial sample counts. Since evaluation of potential differences between manufacturing processes $\mathrm{A}$ and $\mathrm{B}$ was the primary objective of the study, matched time points were selected across manufacturing processes $\mathrm{A}$ and $B$ within each subject so that differences between the processes could be evaluated in an qualitative visual manner, even though not powered or balanced for a formal statistical evaluation.

\section{Calculations for incurred sample reproducibility} As this experiment was not a pure ISR design due to the elapsed time, reagent changes, and low concentration as noted above, a formal statistical determination for the acceptance criteria was not determined. The samples selected for this cell-based assay were generally lower concentration, with nearly a third below the $3 \times$ LLOQ threshold typically used for ISR sample selection. Using the $30 \%$ variability at the LLOQ relative to the $20 \%$ variability at the analytical QC levels for a typical immunoassay, the $30 \%$ acceptance criterion for immunoassay ISR was multiplied by $50 \%$ to $45 \%$. This simple approach was vetted with the PK scientist and statistician prior to implementation. Due to the intersubject variability typical for Drug $\mathrm{X}$, this approach was acceptable in this case.

Assay variability was calculated as follows:

$$
\text { Assay variability }(\%)=\frac{(\text { First result }- \text { ISR result }) \times 100}{(\text { First result }+ \text { ISR result }) / 2}
$$

Equation 1

With this formula, an ISR result lower than the reported result yields a positive assay variability percentage and an ISR result higher than the reported result yields a negative assay variability percentage. 
Although not part of the acceptance criteria for the ISR, additional summary statistics were calculated using Excel 2010 (Microsoft, Seattle, WA) from the individual sample assay variability results. The median and mean $\%$ variability was calculated across all individual samples, all process $\mathrm{A}$ samples and all process $\mathrm{B}$ samples. In addition, the standard deviation and 95\% confidence interval around the mean were calculated using a normal distribution.

\section{Results \& discussion}

Overall results of incurred sample reproducibility The incurred sample reproducibility (Figure 1) passed the pre-established acceptance criteria: 174/216 samples $(80.6 \%)$ were within $45 \%$ of the original valid result. The lowest assay variability that 144/216 samples $(67 \%)$ met was $37 \%$. The results are rank ordered with the highest original concentration on the left and the lowest original concentration on the right; the dashed vertical line marks the samples at $3 \times$ LLOQ. There was no clear trend in the assay variability with concentration, although there were proportionally more excursions at the concentrations near $2 \times$ LLOQ (furthest right samples). If the analysis was restricted to samples $\geq 3 \times$ LLOQ, a more typical ISR sample selection criterion, $124 / 146$ samples (84.9\%) were within $45 \%$ of the original valid result.

As the arithmetic mean of the assay variability was $-29.0 \%$ (Table 1), the stability of incurred samples was confirmed as expected based on the prestudy validation with spiked QCs. The negative percentage indicates that the values obtained during the ISR were on average higher than the valid reported values, suggesting that there was no loss in sample integrity relative to the original result. This is consistent with expectations and scientific assessment that this assay was unlikely to have differential stability between spiked and incurred samples.

\section{Subanalysis by manufacturing process}

A qualitative analy/sis was performed on the subsets of samples across each manufacturing process from



Figure 1. Assay variability from incurred sample reproducibility, rank ordered with the highest original result concentrations on the left and the lowest original result concentrations on the right. The dotted lines are the acceptance criterion of $\pm 45 \%$. The vertical line represents the sample with original concentration equal to $3 \times$ LLOQ. Negative percent variability indicates a higher incurred sample reproducibility concentration than the reported result. 
Table 1. Statistics for individual\% assay variability calculations across samples.

\begin{tabular}{|llll|}
\hline Statistic & All samples & Process A samples & Process B samples \\
\hline Mean ${ }^{\dagger}$ & -29.0 & -31.1 & -26.5 \\
\hline Median & -29.7 & -32.4 & -24.1 \\
\hline $\mathrm{SD}^{+}$ & 20.2 & 20.7 & 19.4 \\
\hline $95 \% \mathrm{Cl}^{+}$ & -26.3 to -31.7 & -27.2 to -35.1 & -22.8 to -30.2 \\
\hline${ }^{+}$Negative percent variability indicates a higher incurred sample reproducibility concentration than the reported result. \\
\hline
\end{tabular}

the cross-over bioequivalence design by graphing the results from manufacturing processes $A$ and $B$ separately (Figure 2). To visually enable the alignment across manufacturing processes $\mathrm{A}$ and $\mathrm{B}$ within a subject, these samples are rank ordered on the $\mathrm{X}$ axis by subject ID and time point rather than by concentration. Qualitatively, the graphs for manufacturing processes A and B show similar assay variability and assay reproducibility. The summary statistics (Table 1) demonstrated overlapping confidence intervals for all samples versus the two sample subsets from each manufacturing process. The similar performance for samples from both arms of the crossover design provided additional confidence that the net bias in the ISR analysis did not adversely affect the final study conclusion.

\section{Conclusion}

By considering the intended purpose of the study and the assay performance characteristics from prestudy validation, an appropriate ISR experiment was designed with pre-established ISR acceptance criteria. Using an assay with wider accuracy and precision acceptance criteria than the regulatory guidances is a fit-for-purpose assessment. Once the assay is selected for that study, using a proportional percentage threshold to set ISR acceptance criterion worked as an appropriate starting point in this case study, given the assay variability at the ISR sample concentrations, interobject variability accounted for in the subject number during bioequivalence study design, and confounding the ISR experiment by conducting nearly two years after original analysis. By expecting the ISR experiment to replicate the accuracy and precision of the original validation, the goal of demonstrating equivalent performance was met.

This ISR experiment demonstrated that the cellbased assay provided reproducible Drug X concentration data. Due to the elapsed time between original and repeat testing, this experiment also supported stability being similar to the pre-established stability with spiked QCs. This finding further supports the anecdotal evidence in the field that spiked sample stability is generally representative of incurred sample stability for biological products (personal communications with members of AAPS Ligand Binding Assay Focus Group). The acceptable ISR and stability results further support the original study conclusion of bioequivalence between manufacturing processes $\mathrm{A}$ and $\mathrm{B}$.



Figure 2. Subanalysis for incurred sample reproducibility. Manufacturing process (A) A and (B) B. The samples are ordered by subject number and time point to display the matched samples from the cross-over design in the same position against the $X$-axis in both Figure $2 \mathrm{~A}$ and $2 \mathrm{~B}$. The dotted lines are the acceptance criterion of $\pm 45 \%$. Negative percent variability indicates a higher incurred sample reproducibility concentration than the reported result. 


\section{Future perspective}

As the diversity of biotherapeutics continues to grow, including novel moieties, the assays measuring drug concentration will also evolve. In addition to this case study, other drug development programs across the industry may use a variety of nonimmunoassay formats for determining drug concentration - such as enzymatic, immunohistochemistry, flow cytometry and other cell-based assays. These assays are selected based on the best balance of assay performance characteristics for the measurement of the individual biotherapeutic, which may not always be the most precise format. As more gene and stem cell therapies advance through clinical development, the assay formats are expected to continue to evolve faster than regulatory and industry guidance. This case study illustrates one approach to adapting ISR principles applied for immunoassay formats to address cell-based assays.

Although a simple proportional method was used for this case study, more rigorous statistical methods can be applied [10-11]. For new drug development programs without known intersubject variability, these statistical models should be investigated as appropriate for fit-for-purpose drug concentration assays.

In addition to technological advancement, regulatory recommendations also continue to evolve based on the aggregate body of data presented by sponsors. This may necessitate additional in-study validation experiments and analysis after the conclusion of primary testing to adequately support a filing. By carefully considering all of the confounding factors, mitigating risk where possible, and considering ways to evaluate the root cause of a potential validation parameter failure, the bioanalytical scientist can conduct a post hoc experiment to satisfy the scientific question from regulators and support the validity of the original conclusion.

\section{Financial \& competing interests disclosure}

The authors of this paper all are, or were, employees of Biogen, which paid for this study to be conducted. The authors have no other relevant affiliations or financial involvement with any organization or entity with a financial interest in or financial conflict with the subject matter or materials discussed in the manuscript apart from those disclosed.

No writing assistance was utilized in the production of this manuscript.

\section{Ethical conduct of research}

The authors state that they have obtained appropriate institutional review board approval or have followed the principles outlined in the Declaration of Helsinki for all human or animal experimental investigations. In addition, for investigations involving human subjects, informed consent has been obtained from the participants involved.

\section{Open access}

This work is licensed under the Creative Commons Attribution-NonCommercial 3.0 Unported License. To view a copy of this license, visit http://creativecommons.org/licenses/bync-nd/3.0/

\section{Executive summary}

- Incurred sample reproducibility acceptance criteria for different assay formats including cell-based assays can be adapted from the general principles for chromatographic assays and immunoassays.

- As time elapses between original and repeat tests, an incurred sample reproducibility experiment has the potential to blend with stability.

- Designing an incurred sample reproducibility experiment for unusual circumstances requires consideration of the study purpose and role of the assay in supporting study conclusions.

\section{References}

1 Viswanathan CT, Bansal S, Booth B et al. Workshop/ Conference Report - Quantitative bioanalytical methods validation and implementation: best practices for chromatographic and ligand binding assays. AAPS J. 9(1), E30-E42 (2007).

2 Fast DM, Kelley M, Viswanathan CT et al. Workshop Report and Follow-up - AAPS workshop on current topics in GLP bioanalysis: assay reproducibility for incurred samplesimplications of crystal city recommendations. AAPS J. 11(2), 238-241 (2009).

3 Committee for Medicinal Products for Human Use (CHMP). Guideline on bioanalytical method validation. EMEA/CHMP/EWP/192217/2009.

www.ema.europa.eu
4 US Department of Health and Human Services, Food and Drug Administration, Center for Drug Evaluation and Research (CDER), Center for Biologics Evaluation and Research (CBER). Draft guidance for industry: bioanalytical method validation (2013).

www.fda.gov

5 Wei X, Grill DS, Heatherington AC et al. Development and validation of a quantitative cell-based bioassay for comparing the pharmacokinetic profiles of two recombinant erythropoietic proteins in serum. J. Pharm. Biomed. Anal. 43(2), 666-76 (2007).

6 van de Merbel N, Savoie N, Manish M et al. Stability assessment in quantitative bioanalysis - recommendations from the Global Bioanalysis Consortium. AAPS J. 16, 392-399 (2014). 
7 DeSilva B, Smith W, Weiner R et al. Recommendations for the bioanalytical method validation of ligand-binding assays to support pharmacokinetic assessments of macromolecules. Pharm. Res. 20(11), 1885-1900 (2003).

8 International Conference on Harmonisation of Technical Requirements for Registration of Pharmaceuticals for Human Use. ICH harmonized tripartite guideline guideline for good clinical practice: E6(R1). International Conference on Harmonisation of Technical Requirements for Registration of Pharmaceuticals for Human Use. www.ich.org
9 Declaration of Helsinki - ethical principles for medical research involving human subjects. World Medical Association. www.wma.net

10 Rocci ML, Devanarayan V, Haughey DB, Jardieu P. Confirmatory reanalysis of incurred bioanalytical samples. AAPS J. 9(3), E336-E343 (2007).

11 Hoffman D. Statistical considerations for assessment of bioanalytical incurred sample reproducibility. AAPS J. 11(3), 570-580 (2009). 